\title{
MAGI-1, A Candidate Stereociliary Scaffolding Protein, Associates with the Tip-Link Component Cadherin 23
}

\author{
Zhigang Xu, ${ }^{1}$ Anthony W. Peng, ${ }^{1,2}$ Kazuo Oshima, ${ }^{1}$ and Stefan Heller ${ }^{1}$ \\ ${ }^{1}$ Departments of Otolaryngology - Head and Neck Surgery and Molecular and Cellular Physiology, Stanford University School of Medicine, Stanford, \\ California 94305, and 2Program in Health Sciences and Technology—Speech and Hearing Bioscience and Technology, Harvard-Massachusetts Institute of \\ Technology, Cambridge, Massachusetts 02139
}

Inner ear hair-cell mechanoelectrical transduction is mediated by a largely unidentified multiprotein complex associated with the stereociliary tips of hair bundles. One identified component of tip links, which are the extracellular filamentous connectors implicated in gating the mechanoelectrical transduction channels, is the transmembrane protein cadherin 23 (Cdh23), more specifically, the haircell-specific Cdh23 $(+68)$ splice variant. Using the intracellular domain of Cdh23 $(+68)$ as bait, we identified in a cochlear cDNA library MAGI-1, a MAGUK (membrane-associated guanylate kinase) protein. MAGI-1 binds via its PDZ4 domain to a C-terminal PDZ-binding site on Cdh23. MAGI-1 immunoreactivity was detectable throughout neonatal stereocilia in a distribution similar to that of Cdh23. As development proceeded, MAGI-1 occurred in a punctate staining pattern on stereocilia, which was maintained into adulthood. Previous reports suggest that Cdh23 interacts via an internal PDZ-binding site with the PDZ1 domain of the stereociliary protein harmonin, and potentially via a weaker binding of its $\mathrm{C}$ terminus with harmonin's PDZ2 domain. We propose that MAGI-1 has the ability to replace harmonin's PDZ2 binding at Cdh23's C terminus. Moreover, the strong interaction between PDZ1 of harmonin and Cdh23 is interrupted by a 35 aa insertion in the hair-cell-specific Cdh23(+68) splice variant, which puts forward MAGI-1 as an attractive candidate for an intracellular scaffolding partner of this tip-link protein. Our results consequently support a role of MAGI-1 in the tip-link complex, where it could provide a sturdy connection with the cytoskeleton and with other components of the mechanoelectrical transduction complex.

Key words: cochlea; hair cell; inner ear; mechanotransduction; protocadherin 15; Usher syndrome

\section{Introduction}

Cadherin 23 (Cdh23) is an atypical cadherin protein with up to 27 extracellular cadherin repeats, a transmembrane domain, and a short cytoplasmic domain. Mutations in the human $\mathrm{CDH} 23$ gene cause Usher (USH) syndrome, which is defined by the association of hearing loss with retinitis pigmentosa (Gorlin et al., 1995). Nine USH genes have been identified to date. It has been shown that most USH proteins localize to the stereocilia of sensory hair cells, where many of them are able to interact with each other (Adato et al., 2005a; El-Amraoui and Petit, 2005).

The murine $C d h 23$ gene gives rise to several splice variants, encoding proteins with different numbers of extracellular cadherin repeats (Lagziel et al., 2005). A second type of alternative splicing of the Cdh23 gene involves the inclusion of exon 68, which encodes part of the cytoplasmic domain of the Cdh23 $(+68)$ isoform that is preferentially expressed in the inner

Received Aug. 12, 2008; revised Sept. 21, 2008; accepted Sept. 24, 2008.

This work was supported by National Institutes of Health Grant DC04563. We thank Drs. A. Ricci and A. Cheng for critically reading this manuscript and members of our research group for helpful discussions, S. Mann for expert technical help, and Dr. C. E. Corrales for help with immunohistochemistry. We thank Dr. K. Noben-Trauth (National Institute on Deafness and Other Communication Disorders, Rockville, MD) for mouse (dh23 cDNA, Dr. K. M. Patrie (University of Michigan, Ann Arbor, MI) for mouse MAGI-1 CDNA, and Dr. U. Müller (Scripps Research Institute, La Jolla, CA) for antibody to (dh23.

Correspondence should be addressed to Dr. Stefan Heller, 801 Welch Road, Stanford, CA 94305. E-mail: hellers@stanford.edu.

DOI:10.1523/JNEUROSCI.3833-08.2008

Copyright $\odot 2008$ Society for Neuroscience $\quad 0270-6474 / 08 / 2811269-08 \$ 15.00 / 0$ ear (Di Palma et al., 2001b), where it has been reported as the exclusive isoform expressed by hair cells (Siemens et al., 2004). It has been suggested that Cdh23(+68), in conjunction with the USH protein protocadherin 15 (Pcdh15), forms tip links (Siemens et al., 2004; Kazmierczak et al., 2007), which are filamentous connectors of the tips of shorter stereocilia with the sides of their longer neighboring stereocilia. Tip links play a crucial role in hair-cell mechanotransduction by translating mechanical forces that lead to gating of the transduction channels. Moreover, in neonatal hair cells, Cdh23 is also part of the transient lateral stereociliary and kinociliary links (Michel et al., 2005; Kazmierczak et al., 2007).

The internal PDZ domain-binding site of Cdh23's intracellular domain interacts with the PDZ1 domain of harmonin. A weaker second interaction site of these two proteins involves Cdh23's C-terminal PDZ domain-binding site and PDZ2 of harmonin (Boëda et al., 2002; Siemens et al., 2002). The internal PDZ domain binding site in Cdh23 is disrupted in the hair-cellspecific Cdh23 $(+68)$ isoform, which leads to starkly reduced general binding of harmonin to this isoform when compared with that of the more widely expressed Cdh23(-68) variant (Siemens et al., 2002). Because the Cdh23 $(+68)$ isoform has been put forward as part of the tip-link complex (Siemens et al., 2004; Kazmierczak et al., 2007), we speculated that other proteins, beside harmonin, might provide a more robust scaffolding network for the Cdh23 $(+68)$ intracellular domain in hair-cell stereocilia. 
To build on this hypothesis, we conducted yeast two-hybrid screens of a cochlear cDNA library with the Cdh23 $(+68)$ intracellular domain. We isolated MAGI-1, a member of the membrane-associated guanylate kinase (MAGUK) protein family (Dobrosotskaya et al., 1997) that contains 6 PDZ domains, 2 WW domains, and 1 guanylate kinase-like (GuK) domain, making it an ideal scaffold for organizing multiple proteins into a protein complex. MAGIs display an inverted domain organization when compared with other MAGUKs, which is reflected in the name MAGI: MAGUKs with Inverted orientation. Three MAGIs have been identified, MAGI-1, MAGI-2, and MAGI-3. Here we show that only MAGI-1, but not MAGI-2 or MAGI-3, binds via its PDZ4 domain to the C-terminal PDZ domain-binding site of Cdh23(-68) and Cdh(+68). By immunolabeling, we show that, like Cdh23, MAGI-1 is localized throughout neonatal hair-cell stereocilia and in a punctate staining pattern in older specimens. Our data suggests that MAGI-1 is an ideal candidate for association with the $\mathrm{C}$ terminus of Cdh23 beneath the stereociliary plasma membrane of interstereociliary links.

\section{Materials and Methods}

Yeast two-hybrid screen. The yeast two-hybrid screen was performed as described previously (Cuajungco et al., 2006) with the following modifications. For bait plasmid construction, we PCR amplified cDNA encoding the C-terminal 265 aa of chicken cdh23 $(+68)$ from chicken cochlear cDNA, cloned it in-frame into vector $\mathrm{pBD}-\mathrm{GAL} 4$ Cam (Stratagene), and verified the sequence. The yeast strain AH109 (Clontech), transformed with this bait plasmid, was then transformed with a chicken basilar papilla cDNA library in the HybriZAP two-hybrid vector (Heller et al., 1998). HIS3 was used as the reporter gene for the screen, and its autoactivation was suppressed with $2.5 \mathrm{~mm} 3$-amino-1,2,4-triazole. Five million transformants were selectively screened, and positive colonies were tested for activation of two other reporter genes, ADE2 and lacZ. The pAD-GAL4-based phagemid vectors in triple-positive yeast colonies were recovered, and cDNA inserts were sequenced. The cDNA encoding for PDZ2-PDZ5 of chicken MAGI-1 was used for reversed yeast twohybrid screen according to the same protocol. A total of $2 \times 10^{6}$ transformants were screened in the presence of $10 \mathrm{~mm} 3$-amino-1,2,4-triazole.

Expression vectors. All cDNA sequences described here are mouse unless indicated otherwise. HA-MAGI-1c and Myc-MAGI-1c are gifts from K. M. Patrie (University of Michigan, Ann Arbor, MI). Different domains of MAGI-1c were subcloned into pEGFP-C2 as follows: PDZ0 (1-110 aa), GuKc (106-300 aa), WW (296-440 aa), PDZ1 (441-620 aa), PDZ2 (621-814 aa), PDZ3 (815-960 aa), PDZ4 (961-1100 aa), PDZ5 (1101-1232 aa), and Cter (1232-1471 aa). Myc-MAGI-1c $\Delta$ PDZ4 that encodes Myc-tagged MAGI-1c without PDZ4 (961-1100 aa) was constructed using PCR. Myc-Cdh23 is a gift from K. Noben-Trauth (National Institute on Deafness and Other Communication Disorders, Rockville, MD) and consists of Cdh 23 cDNA encoding for the first three extracellular cadherin (EC) repeats (1-348 aa) fused to cDNA encoding the protein's C terminus [2975-3354 aa in Cdh23 (+68), 2975-3319 aa in
Cdh23(-68)] including the transmembrane domain. For protein manipulation, we added His and c-Myc tags between the signal peptide and the first EC repeat. The cDNA encoding for the cytoplasmic domain of Cdh23 was PCR amplified and cloned into pHA-RK5 to generate an expression vector for HA-Cdh23-Cter. The cDNA encoding for the last 108 aa (or 104 aa, missing the last 4 aa, "ITEL") at the C terminus of Cdh23 was PCR amplified and cloned into pcDNA3.1(+) to generate expression vectors for Myc-Cdh23-Cend and Myc-Cdh23-Cend(-ITEL). Harmonin and MAGI2-PDZ4 (888-1056 aa), and MAGI3-PDZ4 (825$977 \mathrm{aa}) \mathrm{cDNAs}$ were amplified from mouse organ of Corti cDNA and cloned into pcDNA3.1(+). Myc-MAGI-2 (rat) cDNA is a gift from X. Zha (Fudan University, Shanghai, China) and MAGI-3-His (human) cDNA is a gift from Y. Hata (Tokyo Medical and Dental University, Tokyo, Japan).

Coimmunoprecipitation. HEK293 cells were transfected with the expression vectors using GeneJammer transfection reagent (Stratagene). Transfected cells were washed with PBS 24-48 h after transfection and lysed in ice-cold lysis buffer consisting of $150 \mathrm{~mm} \mathrm{NaCl}, 50 \mathrm{~mm}$ Tris at $\mathrm{pH}$ 7.5, 1\% (v/v) Triton X-100, $1 \mathrm{~mm}$ PMSF, and $1 \times$ protease inhibitor cocktail (Sigma-Aldrich). For immunoprecipitation, we used immobilized monoclonal anti-c-Myc agarose beads (Pierce) and performed the 


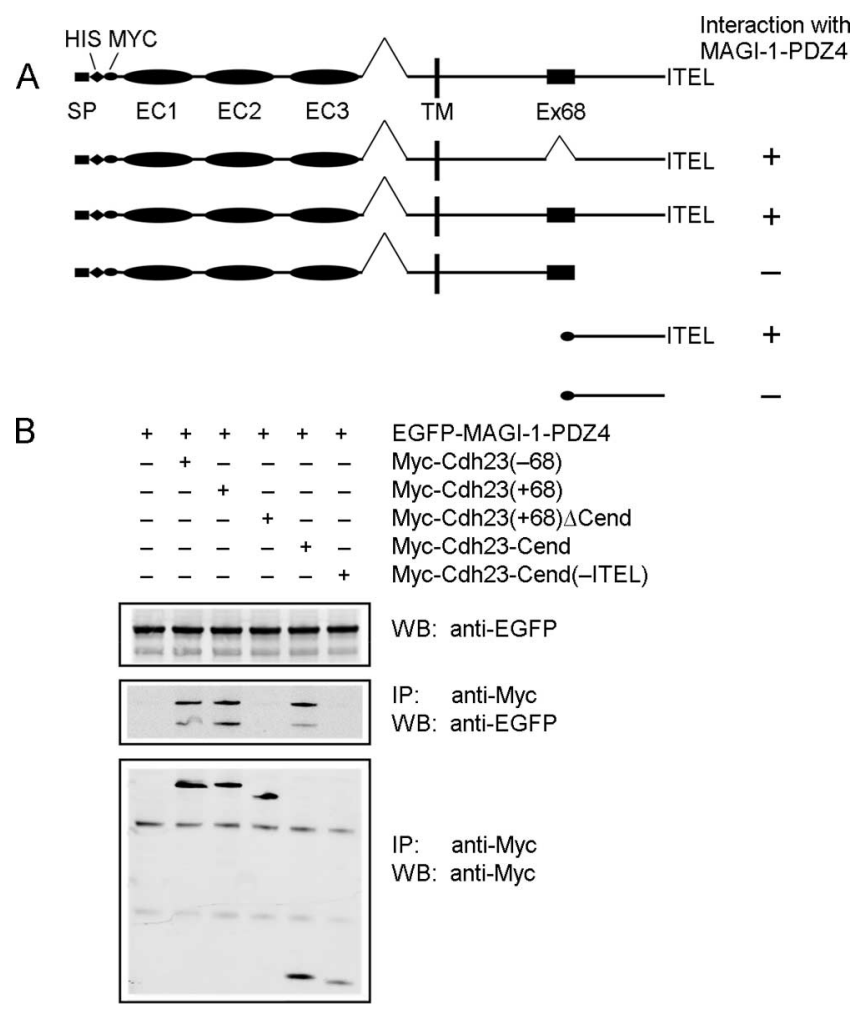

Figure 3. Cdh23's four C-terminal amino acids are essential for MAGI-1 binding. $A$, Schematic drawing of the different variants of $\mathrm{Cdh} 23$ that we used for coimmunoprecipitation experiments. $\boldsymbol{B}$, Western blots showing lack of coimmunoprecipitation of MAGI-1's PDZ4 domain in absence of all C-terminal amino acids after exon 68 [(Myc-Cdh23 $(+68 \Delta$ Cend)] as well as in the absence of only the last four C-terminal amino acids [(Myc-Cdh23 Cend(-ITEL)].

experiments according to the manufacturer's recommendation. After $2 \mathrm{~h}$ of incubation at $4^{\circ} \mathrm{C}$, immunoprecipitated proteins were washed five times with washing buffer (a modified lysis buffer containing $500 \mathrm{~mm}$ $\mathrm{NaCl}$ instead of $150 \mathrm{~mm}$ ), separated by PAGE, then transferred to nitrocellulose membrane and detected with an Odyssey Infrared Imaging System (LI-COR Biosciences).

$R T-P C R$. Total RNA was isolated from postnatal day 5 mouse (C57BL/6) tissues using RNeasy Micro Kits (Qiagen) and reversetranscribed into cDNA using SuperScript III (Invitrogen) with oligo-dT. Polymerase chain reaction was performed using this cDNA as the template with the following primers: $\beta$-actin sense, $5^{\prime}$-ACGGCCAGGTCATCACTATTG-3', antisense, 5'-AGGGGCCGGACTCATCGTA-3' (372bp); MAGI-1a sense, 5'-GTCTTCGAGGGGGCCGAGAATATAACATGG-3', antisense, 5'-TGGAGTCATGCCAGGGAAGGAAG-3' (277bp); MAGI-1b/c sense, 5'-GTCTTCGAGGGGGCCGAGAATATAACATGG-3', antisense, 5'-GGTGGAGGGGCCGTTCCTGTCG-3' (1b variant $349 \mathrm{bp}$, 1c variant 260bp); Cdh23 sense, 5'-GACAACATCGCCAAGCTG-3', antisense, 5'-GCAAGCTGTTGAGATCAGTGG-3' (+68 variant $316 \mathrm{bp},-68$ variant $211 \mathrm{bp}$ ). To achieve the best possible sensitivity and specificity, we adjusted cycle lengths for different PCR reaction sets to between 30 and 33 cycles, and annealing temperatures to between 60 and $62^{\circ} \mathrm{C}$.

Whole-mount immunocytochemistry. All animal procedures followed guidelines set forth by the National Institutes of Health. Samples of organ of Corti from $\mathrm{BALB} / \mathrm{c}$ mice were fixed with cold $4 \%$ paraformaldehyde in PBS for $30 \mathrm{~min}$, then permeabilized and blocked with PBT1 (0.1\% Triton $\mathrm{X}-100,1 \% \mathrm{BSA}, 5 \%$ heat-inactivated goat serum in PBS at $\mathrm{pH} 7.3$ ) for 30 min. All steps were performed at room temperature unless otherwise indicated. Samples were incubated overnight at $4^{\circ} \mathrm{C}$ with $20 \mu \mathrm{g} / \mathrm{ml}$ rabbit anti-MAGI-1 antibody (H-70, Santa Cruz Biotechnology), or rabbit anti-Cdh23 antibody raised against amino acids 3,133-3,291, and affinity purified using a peptide with the amino acid sequence encoded by exon 68 (Siemens et al., 2004) a gift from U. Müller (The Scripps Research
Institute, La Jolla, CA). The commercially available MAGI-1 antibody was raised against the $\mathrm{N}$-terminal 70 aa. We confirmed the MAGI-1 antibody specificity by Western blot (supplemental Fig. S1, available at www.jneurosci.org as supplemental material) and by immunolocalization of an EGFP-MAGI-1 fusion protein expressed in HEK293 (Fig. S2, available at www.jneurosci.org as supplemental material) and LLC-PK1 cells (data not shown), where the immunostaining completely colocalized with the green fluorescence. A control EGFPMAGI-1 fusion protein lacking PDZ0 (amino acids 1-110) was not immunostained by MAGI-1 antibody (Fig. S2, available at www. jneurosci.org as supplemental material). For blocking, we used a combination of three overlapping MAGI-1 peptides covering amino acids $1-30,21-50$, and $41-70$ at a final concentration of $300 \mu \mathrm{g} / \mathrm{ml}$ per peptide. The antibody was preincubated in these peptides for $2 \mathrm{~h}$ before immunodetection. We used two additional antibodies: monoclonal anti-MAGI-1 (cat\# H0009223-M03; Novus Biologicals) and rabbit anti-MAGI-1 (cat\# M5691; Sigma) to confirm MAGI-1 immunostaining. Both antibodies required a 15 min-treatment with $1 \%$ SDS in PBS before blocking, and overnight incubation of the primary antibody at $30^{\circ} \mathrm{C}$, which resulted in poor stereociliary morphology. After primary antibody staining, the samples were washed twice with PBT1 for 10 min and twice with PBT2 (0.1\% Triton X-100, 0.1\% BSA in PBS) for $5 \mathrm{~min}$, and incubated with $7.5 \mu \mathrm{g} / \mathrm{ml}$ FITC-conjugated goat anti-rabbit antibody (Jackson ImmunoResearch) in PBT2 for $2 \mathrm{~h}$, followed by two $5 \mathrm{~min}$ PBT2 washes. Samples were incubated with $4 \mu \mathrm{g} / \mathrm{ml}$ TRITC-conjugated phalloidin in PBS for $20 \mathrm{~min}$, followed by three 5 min washes with PBS, then mounted in Cytomation fluorescent mounting medium (Dako) and imaged with a confocal microscope (LSM Pascal; Zeiss).

\section{Results}

\section{MAGI-1 is a Cdh23 interaction partner}

To identify Cdh23 interaction partners, we carried out yeast twohybrid screens of a chicken basilar papilla cDNA library using the intracellular part of chicken Cdh23 $(+68)$ protein as a bait. We noticed a strong interaction with a cDNA library clone encoding the chicken MAGUK protein MAGI-1 (GenBank accession number XR_027096). MAGI-1 occurs in three splice variants: 1a, 1b, and 1c, which have different $\mathrm{C}$ termini (Dobrosotskaya et al., 1997). The cDNA that we isolated corresponded to MAGI-1c. In a reversed library screen using a partial chicken MAGI-1c protein encoding PDZ domains $2-5$ as bait, we reciprocally identified a truncated chicken Cdh23 cDNA clone encoding the 213 C-terminal amino acids of the Cdh23 $(+68)$ isoform. The unambiguous identifications attained with yeast two-hybrid screens from a complex pool of millions of yeast transformants in each individual experiment suggested a strong interaction between these two proteins.

For coimmunoprecipitation experiments, we switched species from avian to rodent, and we confirmed the interaction of Cdh23 with MAGI-1c. Myc epitope-tagged murine Cdh23(+68) and Cdh23(-68) coimmunoprecipitated hemagglutinin epitope tag (HA) epitope-tagged full-length murine MAGI-1c (Fig. 1A). Likewise, myc epitope-tagged full length MAGI-1c protein coimmunoprecipitated the cytoplasmic domains of both HA epitopetagged Cdh23 $(+68)$ and $\mathrm{Cdh} 23(-68)$ (Fig. 1B). These results confirmed the initial yeast two-hybrid findings and show the interaction between MAGI-1c and Cdh23 are independent of the exon 68 splice variant.

\section{PDZ4 of MAGI-1 and the C-terminal PDZ domain-binding site of $\mathrm{Cdh} 23$ mediate the interaction between the two proteins}

To elucidate the specific region of MAGI-1c that interacts with Cdh23, we expressed individual domains as EGFP fusion pro- 
teins and examined their interaction with Cdh23 by coimmunoprecipitation. Binding occurred only when the MAGI-1 PDZ4 domain was used, whereas other domains, including the MAGI-1c-specific C terminus, did not interact with Cdh23 (Fig. $2 A, B)$. When we deleted PDZ4 from the full-length MAGI-1c protein, we abolished coimmunoprecipitation with $\mathrm{Cdh} 23$ (Fig. 2C). These experiments show that the interaction between the cytoplasmic domain of Cdh23 and MAGI-1 happens via MAGI-1's PDZ4 domain, and that MAGI1-PDZ4 is both sufficient and essential for this interaction. Our results further confirm that the interaction is independent of exon 68, and that all three MAGI-1 splice variants are potential interaction partners of Cdh23 because they all share identical PDZ4 domains.

We next sought to identify the region of Cdh23 that is essential for binding of MAGI-1. When we truncated Cdh23 directly after exon 68, binding to MAGI-1PDZ4 was abolished (Fig. 3A,B). Conversely, the $\mathrm{C}$ terminus starting directly after exon 68 displayed strong binding to MAGI-1-PDZ4. The last four amino acids of the Cdh23 protein sequence "ITEL" form a class-I PDZ-binding interface (Sheng and Sala, 2001), which has previously been shown to mediate binding to the PDZ2 domain of harmonin (Siemens et al., 2002). Deletion of these four amino acids from Cdh23's C terminus eliminated the interaction with MAGI-1PDZ4 (Fig. 3A,B). We consequently conclude that the four C-terminal amino acids of Cdh23 are essential for binding to MAGI-1.

\section{Neither MAGI-2 nor MAGI-3 are able to bind to Cdh23}

MAGI-1 displays substantial sequence similarity with the two other known MAGI proteins MAGI-2 and MAGI-3. This sequence similarity raised the question of whether the PDZ4 domains of MAGI-2 and MAGI-3, which share $71 \%$ and $61 \%$ identity with the PDZ4 domain of MAGI-1, might also mediate binding with Cdh23 (Fig. $4 A$ ). This possibility was further supported by a study that showed binding of MAGI-1, MAGI-2, and MAGI-3 PDZ4 domains to the Notch ligand Delta (Wright et al., 2004). We conducted coimmunoprecipitation experiments with myc epitope-tagged PDZ4 domains of MAGI-1, MAGI-2, and MAGI-3 in conjunction with HA epitope-tagged Cdh23 C terminus. Robust coimmunoprecipitation occurred only in the presence of MAGI-1 PDZ4, (Fig. $4 B$ ), which suggests that MAGI-1 is the only MAGI protein capable of binding to $\mathrm{Cdh} 23$.

\section{MAGI-1 and harmonin can bind to Cdh23 when both are present}

It has been previously suggested that $\mathrm{Cdh} 23$ and harmonin bind to each other and that the majority of this binding is mediated by the internal PDZ domain-binding site of harmonin, which is interrupted in Cdh23 $(+68)$ isoforms (Siemens et al., 2002). The same study also implicated the C-terminal PDZ-binding site of both Cdh23 isoforms as a harmonin-

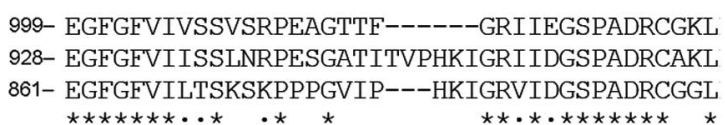

$\star * * * * * * . * . * * * * * . * \cdot * * * * * * *$

KVGDRILAVNGCSITNKSHSDIVNLIKEAGNTVTLRIIPGD -1074 KVGDRILAVNGQSIINMPHADIVKLIKDAGLSVTLRIIPQE -1009 KVGDHISAVNGQSIVDLSHDNIVOLIKDAGVTVTLTVVAEE - 939 $\star * * *: * * * * * *: *^{*}: * *: * * *: * *: * *$ : : : :

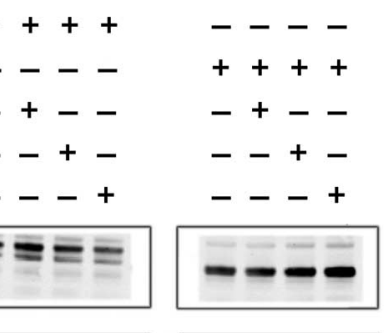

HA-Cdh23(+68)-Cter

HA-Cdh23(-68)-Cter

Myc-MAGI-1-PDZ4

Myc-MAGI-2-PDZ4

Myc-MAGI-3-PDZ4

WB: anti-HA

IP: anti-Myc

WB: anti-HA

IP: anti-Myc

WB: anti-Myc

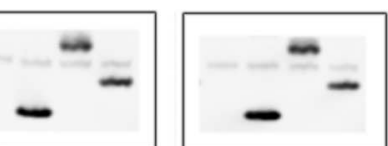

Figure 4. MAGI-1, but not MAGI-2 or MAGI-3, can bind to Cdh23. $\boldsymbol{A}$, Comparison of PDZ4 of MAGI-1 with its closest homologues, PDZ4 of MAGI-2 and MAGI-3. An asterisk indicates identical amino acids in all three sequences, a colon indicates a of HA-tagged Cdh23(+68) and Cdh23(-68) isoforms with MAGI-1's PDZ4 domain, but not with PDZ4 of MAGI-2 or MAGI-3. 


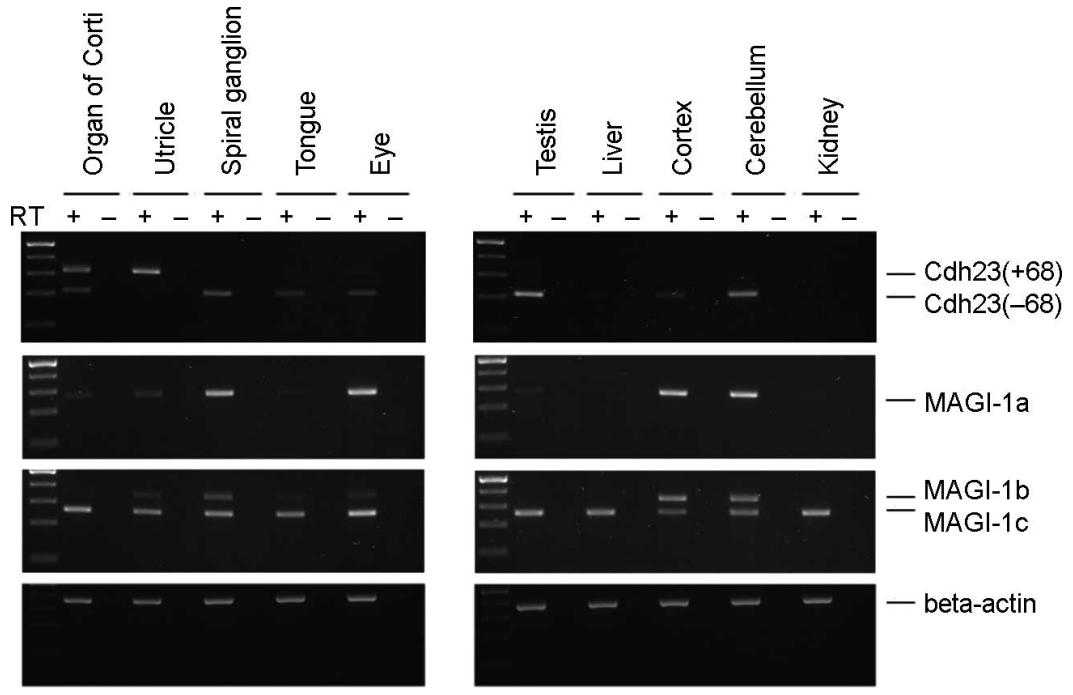

Figure 6. Expression analysis of (dh23 exon 68 and MAGI-1 C-terminal splice variants. Top, Cdh23(+68) mRNA is detectable only in inner ear tissue; $\mathrm{Cdh} 23(-68)$ displays a more wide spread expression. Middle, MAGI-1a and MAGI-1b expression appears to be strongest in nervous tissue, and MAGI-1c is expressed in all tissues/organs investigated. Bottom, $\beta$-Actin specific primers were used for the RT-PCR template control.
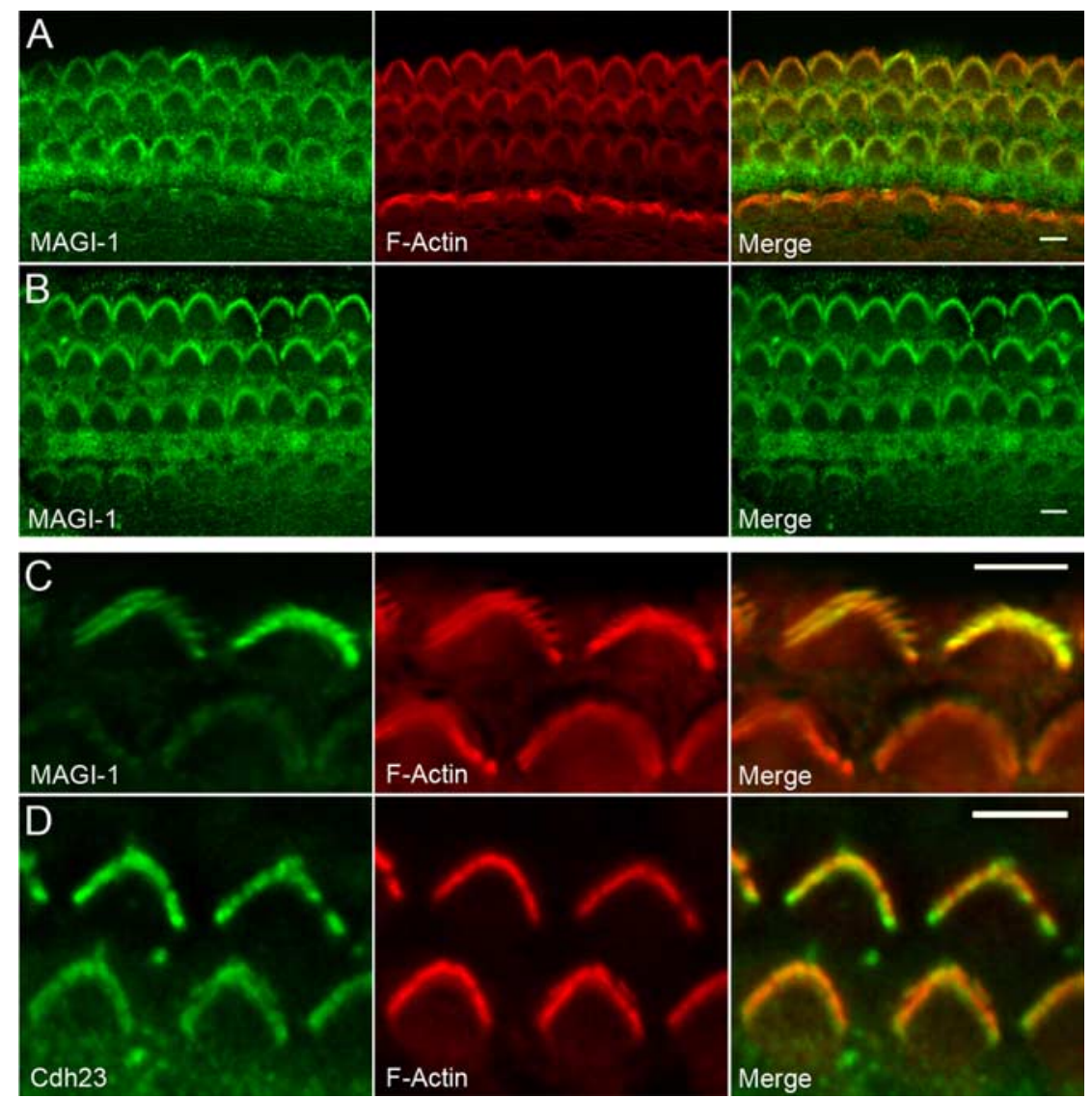

Figure 7. MAGI-1 immunolocalization in P3 mouse cochlear hair cells. Shown are single confocal sections. $\boldsymbol{A}, \mathrm{MAGI}-1$ immunoreactivity visualized with FITC-conjugated secondary antibody is markedly associated with stereocilia, which are visualized with rhodamine-conjugated phalloidin. $\boldsymbol{B}$, Control for the image acquisition procedure omitting rhodamine-conjugated phalloidin in the staining. C, MAGI-1 immunoreactivity is distinctly associated with stereocilia. D, Cdh23 immunoreactivity in the hair bundles of P3 mouse cochlear outer hair cells. Scale bars: $5 \mu \mathrm{m}$.

binding appeared to be weaker, which is in agreement with a previous study (Siemens et al., 2002) and suggestive of a potential preferential interaction of the Cdh23(+68) isoform with MAGI-1.

\section{MAGI-1a and MAGI-1c isoforms are} expressed in the inner ear

The fact that we isolated MAGI-1c from a chicken cochlear cDNA library suggested that this isoform and possibly other MAGI-1 splice variants are expressed in the inner ear. To determine the specific C-terminal MAGI-1 isoforms that occur in the inner ear, we conducted an isoformspecific RT-PCR analysis. We found transcripts representing MAGI-1a and MAGI$1 \mathrm{c}$, but not MAGI-1b, in the mouse organ of Corti (Fig. 6). In the vestibular maculae and in the spiral ganglion, we detected all three known MAGI-1 splice variants. Overall, MAGI-1c was ubiquitously detectable, whereas MAGI- $1 \mathrm{a}$ and MAGI-1b were more restrictively expressed. A parallel analysis of the exon 68 splice variant expression of $\mathrm{Cdh} 23$ confirmed the results of previous reports (Di Palma et al., 2001a; Siemens et al., 2004) that exon 68 is specifically expressed in inner ear sensory epithelia (Fig. 6).

\section{MAGI-1 is a stereociliary protein}

Our RT-PCR analysis showed that MAGI-1 mRNA is detectable in the organ of Corti and the epithelial maculae of the vestibular system. In addition, a recent publication identified MAGI-1 transcripts in purified zebrafish hair cells (McDermott et al., 2007). Based on this information, we hypothesized that MAGI-1 is present in murine hair cells, and we consequently focused on testing whether MAGI-1 protein is detectable in stereocilia, which is where one would expect to find a protein that interacts with the stereociliary protein Cdh23.

In whole-mount preparations of postnatal day 3 organs of Corti, an antibody to MAGI-1-labeled hair-cell stereocilia (Fig. $7 A$ ). Inner hair-cell stereocilia displayed weaker immunolabeling when compared with outer hair-cell stereocilia. At this early neonatal time point, when the hair bundles are still maturing, MAGI- 1 appeared to be located along the stereociliary shafts, as well as in a pattern of stronger punctuate immunofluorescence (Fig. 7C). We also observed MAGI-1 immunoreactivity associated with the disappearing cochlear hair cells' kinocilia, which is not visible in the optical section shown in Figure 7C. Antibodies to $\mathrm{Cdh} 23$ revealed a comparable staining pattern of $\mathrm{P} 3$ cochlear hair cells (Fig. 7D). This Cdh23 distribution is similar to the previously reported localization of Cdh23 in neonatal mouse organ of Corti hair cells (Lagziel et al., 2005; Michel et al., 2005; Phillips et al., 2006; Kazmierczak et al., 2007). In specimens from adult animals, the MAGI-1 immunoreactivity was more 
punctate and detectable in stereocilia of inner as well as outer hair cells (Figs. 8, 9). MAGI-1 immunoreactivity was not restricted to hair-cell stereocilia and was also detectable in junctional complexes in the organ of Corti, presumably tight junctions, and in the cuticular plates of cochlear hair cells (Fig. S3, available at www.jneurosci.org as supplemental material). MAGI-1's stereociliary distribution is compatible with a potential function as a Cdh23 binding partner because Cdh23 immunoreactivity in mature hair cells also appears in a punctate pattern, where it has been shown to be associated with tip links (Kazmierczak et al., 2007; Siemens et al., 2004).

\section{Discussion}

In this study, we identified MAGI-1 as a binding partner of the stereociliary protein Cdh23. We show that the avian and the murine orthologs of the two proteins are able to bind to each other. We mapped the interaction and showed that PDZ4 of all MAGI-1 isoforms is essential and sufficient for binding. For Cdh23, we demonstrated that the protein's intracellular C-terminal region is sufficient for binding MAGI-1, and that the C-terminal amino acids "ITEL," which form a class-I PDZ-binding interface (Sheng and Sala, 2001), are essential for binding to MAGI-1's PDZ4 domain. The interaction between Cdh23 and MAGI-1 is independent of the alternatively spliced exon 68 , which has been shown previously to disrupt a strong binding site for the PDZ1 domain of the Usher protein harmonin (Siemens et al., 2002).

\section{Alternative splicing and the Cdh23-harmonin complex}

It has been proposed that $\mathrm{Cdh} 23$ harbors two independent binding sites for the three-PDZ-domain protein harmonin (Siemens et al., 2002). Site one is located internally in the C-terminal region and binds to harmonin's PDZ1, whereas the four most C-terminal amino acids of Cdh23 make up a second binding site for harmonin, mediated by PDZ2. Binding efficacy at this second site has been approximated at $10 \%$ of the binding efficacy between Cdh23's internal PDZ binding site and harmonin's PDZ1 domain (Siemens et al., 2002). Nevertheless, the binding of harmonin's PDZ2 to Cdh23 has been independently verified (Boëda et al., 2002) and was also consistently detectable in our study. The internal binding site for harmonin's PDZ1 is disrupted in the Cdh23(+68) splice variant expressed by hair cells, which spurred the hypothesis that the presumptive Cdh23-harmonin complex is regulated by alternative splicing. Based on this hypothesis, a model had been put forward in which the predominant retinal Cdh23(-68) isoform interacts strongly with harmonin simultaneously via PDZ1 and PDZ2 (Siemens et al., 2002). In this retinal complex, harmonin's PDZ1 domain is unavailable for self-multimerization. Conversely, the hair-cell Cdh23(+68) isoform cannot bind harmonin's PDZ1, and it has been speculated that a hair- cell Cdh23-harmonin complex consists of Cdh23(+68) variants that bind harmonin via PDZ2. Because this configuration allows for harmonin self-multimerization via PDZ1 and harmonin's C terminus, it would allow the hair-cell Cdh23(+68)-harmonin complex to cluster Cdh 23 proteins. Nevertheless, the interaction between the Cdh23 C terminus and harmonin's PDZ2 has been de- scribed as relatively weak when compared with the interaction with harmonin's PDZ1 (Siemens et al., 2002).

MAGI-1 binds to the Cdh23(-68) and Cdh23(+68) isoforms We hypothesize that MAGI-1, based on its subcellular localization in hair-cell stereocilia and its robust binding characteristics, is an additional, and possibly, an alternative binding partner for Cdh23. For the Cdh23(-68) isoform, three potential configurations are possible in which Cdh23 interacts with harmonin, with MAGI-1 (Fig. 10A), or potentially with both proteins at the same time. We have conducted coimmunoprecipitation experiments to explicitly test for a Cdh23(-68)-harmonin-MAGI-1 complex, but we found no evidence supporting a scenario in which Cdh23 binds harmonin and MAGI-1 simultaneously (data not shown). Based on this, we speculate that harmonin's PDZ1 domain cannot bind well to Cdh23's internal PDZ-binding site when Cdh23's C-terminal binding site is occupied by MAGI-1.

In the alternatively spliced hair-cell-specific configuration, we propose a strong interaction of Cdh23 $(+68)$ with MAGI-1. As proposed previously (Siemens et al., 2002), harmonin's PDZ2 domain is able to bind Cdh23's C terminus, and we found that this interaction is clearly detectable, even in the presence of competing MAGI-1, suggesting that a Cdh23(+68)-harmonin complex could co-exist with a Cdh23(+68)-MAGI-1 complex. This means that Cdh23 $(+68)$ is able to interact either with harmonin or with MAGI-1, but not with both proteins simultaneously (Fig. 10B).

\section{MAGI-1 interacts with the USH protein network and is a} candidate for providing a strong link between the tip link and the cytoskeleton

Cdh23 has been associated with at least three structural elements of hair-cell stereociliary bundles. In developing hair cells, the protein localized to transient lateral links and kinociliary links; in mature hair cells, Cdh23 has been put forward as a component of tip links (Siemens et al., 2004; Lagziel et al., 2005; Michel et al., 2005; Kazmierczak et al., 2007).

The USH protein harmonin has been proposed as the central scaffolding organizer in stereocilia providing a link between the cytoplasmic domains of USH proteins Cdh23, Pcdh15, VLGR1, 

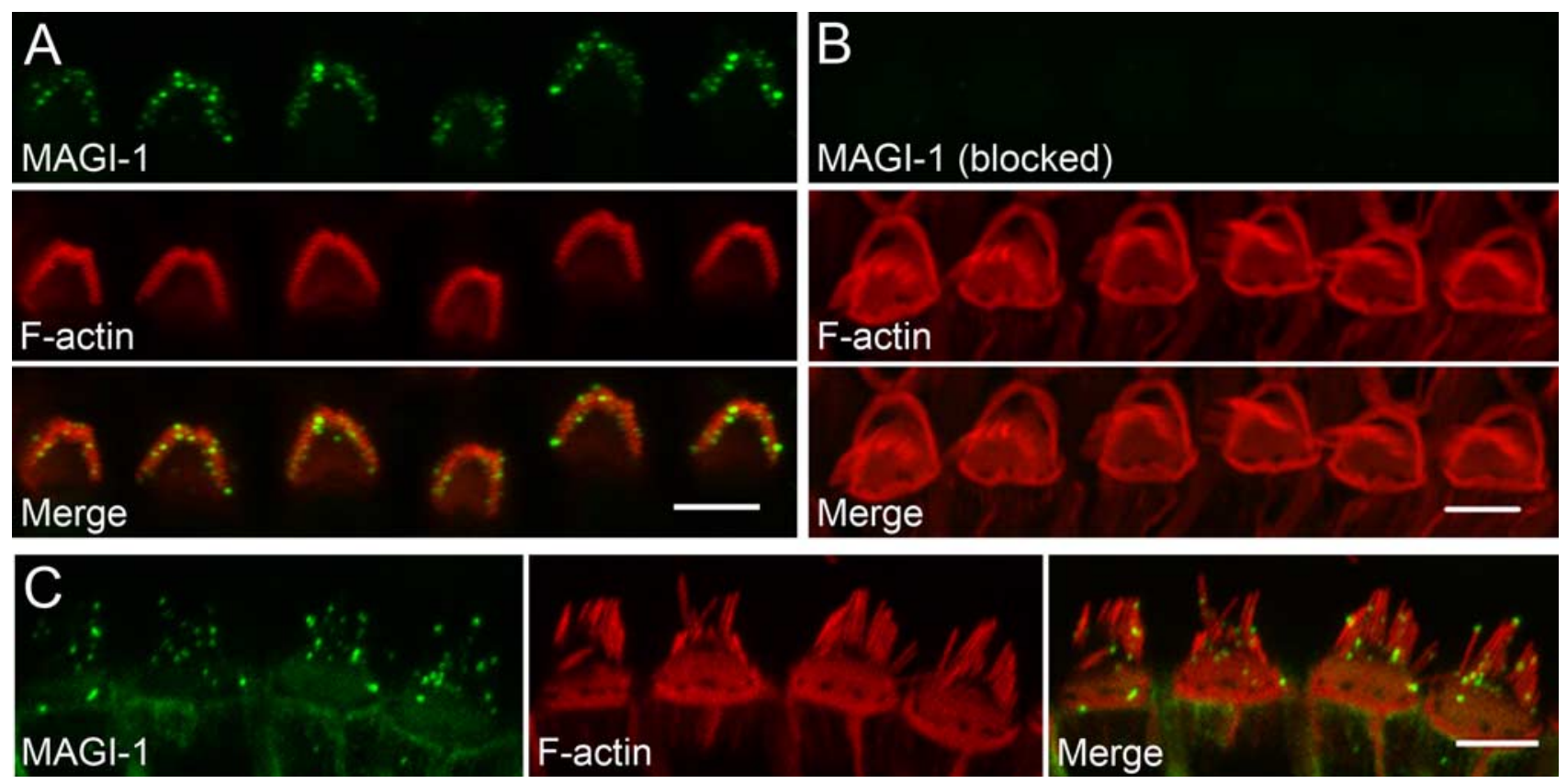

Figure 9. MAGI-1 immunolocalization in the apical turn of 12-week-old mouse cochlear outer hair cells. Shown are maximum image projections of confocal stacks. MAGI-1 immunoreactivity was visualized with FITC-conjugated secondary antibody (green), and stereocilia were visualized with rhodamine-conjugated phalloidin (red). $\boldsymbol{A}$, Top view of stereocilia bundles showing punctate MAGI-1 staining. $\boldsymbol{B}, \mathrm{MAGI}-1$ staining is blocked upon preincubation of the antibody with peptides corresponding to the antigen. C, Splayed outer hair cell stereocilia show a staining pattern similar to that of inner hair cells with punctate staining along the stereocilia. Scale bars: $5 \mu \mathrm{m}$.

and usherin, purportedly with the F-actin core of stereocilia (Adato et al., 2005b; El-Amraoui and Petit, 2005; Reiners et al., 2005). Based on its multiple PDZ-based interactions with many stereociliary proteins, it is well accepted that harmonin fulfills a central stabilizing scaffolding role for USH protein complexes. A recent study reported an association of harmonin with actin filaments, an interaction that could be direct or indirect via an interaction with myosin VIIA (Boëda et al., 2002).

Based on its many protein interaction domains, we hypothesize that MAGI-1 is also an attractive stereociliary scaffolding protein. MAGI-1 is probably not a USH syndrome candidate protein because it displays a more widespread expression pattern than the USH proteins, which are predominantly expressed in the eye and the inner ear. Nevertheless, MAGI-1 has been associated not only with scaffolding functions at sites of cellular attachment including tight and adherens junctions, but also with the postsynaptic orchestration of protein interactions at the differentiating neuromuscular junction (Ide et al., 1999; Mino et al., 2000; Strochlic et al., 2001; Sakurai et al., 2006). Furthermore, it has been proposed that MAGI-1 interacts with $\beta$-catenin, synaptopodin, and $\alpha$-actinin-4 (Dobrosotskaya and James, 2000; Patrie et al., 2002), thereby providing a potential link between the unconventional Cdh23 and the cytoskeleton. Such a link might be important not only during assembly of links, but also in the mature hair bundle when mechanical loads between individual stereocilia have to be conveyed to the actin core of the stereociliary bundle. More recently, caspasemediated cleavage of MAGI-1 has been put forward as an important step for epithelial cell-cell detachment in apoptosis (Gregorc et al., 2007; Ivanova et al., 2007), which indicates that MAGI-1-mediated scaffolding is able to provide a strong mechanical backbone at plasma membrane contact sites.

Our immunocytochemical analysis of MAGI-1 expression demonstrated MAGI-1 immunoreactivity throughout neonatal stereocilia and kinocilia, and in a punctate pattern in stereocilia of adult mice. This distribution is in agreement with an interaction with Cdh23, which is detectable in the neonatal stereocilia and kinocilia, as well as prominent punctate immunolabeling associated with the tip links of mature hair cells in adult animals (Siemens et al., 2004; Lagziel et al., 2005; Michel et al., 2005; Kazmierczak et al., 2007). We tested for antibody specificity by blocking the primary antibody with peptides that cover the antigen used for the immunization. In addition, we have confirmed the immunostaining shown in Figures 8 and 9 with two additional antibodies (data not shown; see Materials and Methods).

We hypothesize that MAGI-1 displays a more efficient binding and is a more suitable scaffolding partner for Cdh23(+68) when compared with harmonin. In this context, we propose that MAGI-1 has the ability to replace harmonin in stereociliary structures that contain the Cdh23 $(+68)$ isoform. MAGI-1 expression persists in mature stereocilia, whereas punctate expression of the ear-specific harmonin b isoform ceases in cochlear hair cells after postnatal day 30, whereas other harmonin isoforms are detectable in the cuticular plate and along the length of stereocilia in older animals (Boëda et al., 2002). Although MAGI-1 was robustly detectable in stereocilia of 12-week-old mice, its punctate immunostaining pattern appeared less organized than the reported Cdh23 immunolabeling of adult cochlear hair cells (Siemens et al., 2004; Kazmierczak et al., 2007).

Based on these considerations, we suggest that MAGI-1 coexists with harmonin during stereociliary development and that it may replace harmonin in some protein complexes in mature stereocilia. Because the Cdh23(+68) isoform has been put forward as a component of stereociliary tip links, we hypothesize that MAGI-1 could be associated with the intracellular domain of Cdh23 (+68) beneath the plasma membrane, at the lateral insertion points of tip links. Here, MAGI-1 might provide a sturdy link with the cytoskeleton and other components of the mature haircell transduction complex. 

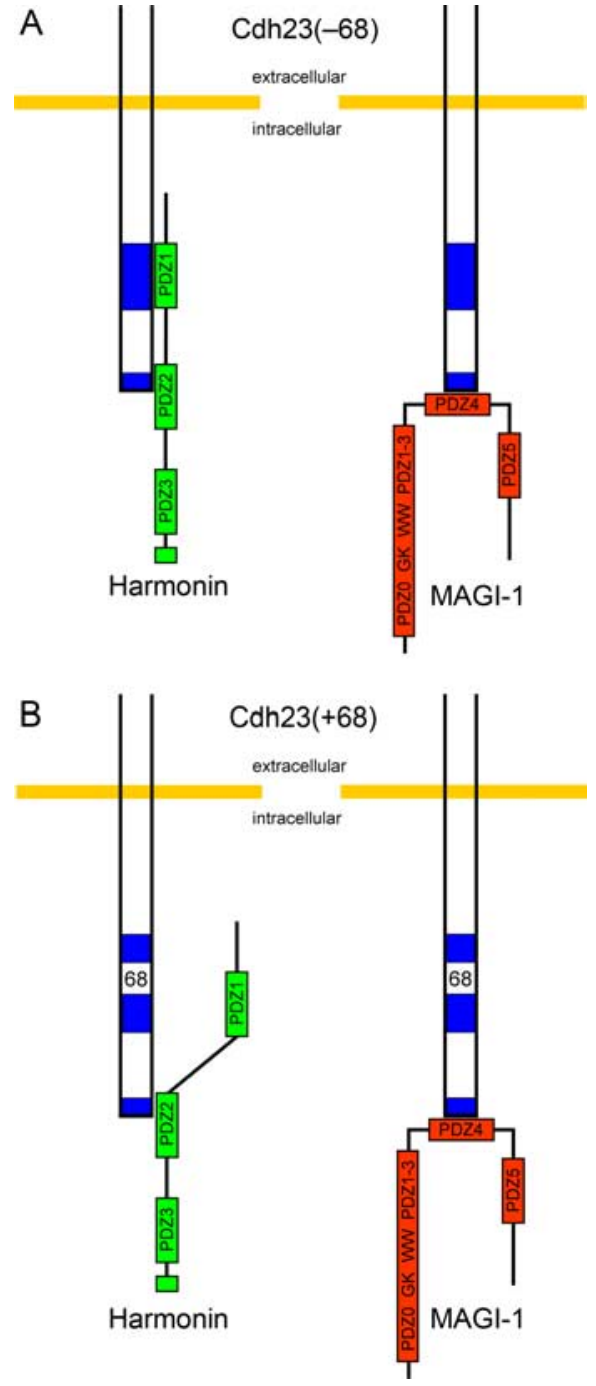

Figure 10. Hypothetical model of binding of $\mathrm{MAGI}-1$ and harmonin to the intracellular domain of $(\mathrm{dh} 23(-68)(A)$ and $C \operatorname{dh} 23(+68)(B)$.

\section{References}

Adato A, Michel V, Kikkawa Y, Reiners J, Alagramam KN, Weil D, Yonekawa H, Wolfrum U, El-Amraoui A, Petit C (2005a) Interactions in the network of Usher syndrome type 1 proteins. Hum Mol Genet 14:347-356.

Adato A, Lefèvre G, Delprat B, Michel V, Michalski N, Chardenoux S, Weil D, El-Amraoui A, Petit C (2005b) Usherin, the defective protein in Usher syndrome type IIA, is likely to be a component of interstereocilia ankle links in the inner ear sensory cells. Hum Mol Genet 14:3921-3932.

Boëda B, El-Amraoui A, Bahloul A, Goodyear R, Daviet L, Blanchard S, Perfettini I, Fath KR, Shorte S, Reiners J, Houdusse A, Legrain P, Wolfrum U, Richardson G, Petit C (2002) Myosin VIIa, harmonin and cadherin 23 , three Usher I gene products that cooperate to shape the sensory hair cell bundle. EMBO J 21:6689-6699.

Cuajungco MP, Grimm C, Oshima K, D’hoedt D, Nilius B, Mensenkamp AR, Bindels RJ, Plomann M, Heller S (2006) PACSINs bind to the TRPV4 cation channel. PACSIN 3 modulates the subcellular localization of TRPV4. J Biol Chem 281:18753-18762.

Di Palma F, Holme RH, Bryda EC, Belyantseva IA, Pellegrino R, Kachar B, Steel KP, Noben-Trauth K (2001a) Mutations in Cdh23, encoding a new type of cadherin, cause stereocilia disorganization in waltzer, the mouse model for Usher syndrome type 1D. Nat Genet 27:103-107.

Di Palma F, Pellegrino R, Noben-Trauth K (2001b) Genomic structure, alternative splice forms and normal and mutant alleles of cadherin 23 (Cdh23). Gene 281:31-41.

Dobrosotskaya I, Guy RK, James GL (1997) MAGI-1, a membrane- associated guanylate kinase with a unique arrangement of protein-protein interaction domains. J Biol Chem 272:31589-31597.

Dobrosotskaya IY, James GL (2000) MAGI-1 interacts with beta-catenin and is associated with cell-cell adhesion structures. Biochem Biophys Res Commun 270:903-909.

El-Amraoui A, Petit C (2005) Usher I syndrome: unravelling the mechanisms that underlie the cohesion of the growing hair bundle in inner ear sensory cells. J Cell Sci 118:4593-4603.

Gorlin RJ, Toriello HV, Cohen MM (1995) Hereditary hearing loss and its syndromes (Oxford monographs on medical genetics). New York/Oxford: Oxford UP.

Gregorc U, Ivanova S, Thomas M, Guccione E, Glaunsinger B, Javier R, Turk V, Banks L, Turk B (2007) Cleavage of MAGI-1, a tight junction PDZ protein, by caspases is an important step for cell-cell detachment in apoptosis. Apoptosis 12:343-354.

Heller S, Sheane CA, Javed Z, Hudspeth AJ (1998) Molecular markers for cell types of the inner ear and candidate genes for hearing disorders. Proc Natl Acad Sci U S A 95:11400-11405.

Ide N, Hata Y, Nishioka H, Hirao K, Yao I, Deguchi M, Mizoguchi A, Nishimori H, Tokino T, Nakamura Y, Takai Y (1999) Localization of membrane-associated guanylate kinase (MAGI)-1/BAI-associated protein (BAP) 1 at tight junctions of epithelial cells. Oncogene 18:7810-7815.

Ivanova S, Repnik U, Banks L, Turk V, Turk B (2007) Cellular localization of MAGI- 1 caspase cleavage products and their role in apoptosis. Biol Chem 388:1195-1198.

Kazmierczak P, Sakaguchi H, Tokita J, Wilson-Kubalek EM, Milligan RA, Müller U, Kachar B (2007) Cadherin 23 and protocadherin 15 interact to form tip-link filaments in sensory hair cells. Nature 449:87-91.

Lagziel A, Ahmed ZM, Schultz JM, Morell RJ, Belyantseva IA, Friedman TB (2005) Spatiotemporal pattern and isoforms of cadherin 23 in wild type and waltzer mice during inner ear hair cell development. Dev Biol 280:295-306.

McDermott BM Jr, Baucom JM, Hudspeth AJ (2007) Analysis and functional evaluation of the hair-cell transcriptome. Proc Natl Acad Sci U S A 104:11820-11825.

Michel V, Goodyear RJ, Weil D, Marcotti W, Perfettini I, Wolfrum U, Kros CJ, Richardson GP, Petit C (2005) Cadherin 23 is a component of the transient lateral links in the developing hair bundles of cochlear sensory cells. Dev Biol 280:281-294.

Mino A, Ohtsuka T, Inoue E, Takai Y (2000) Membrane-associated guanylate kinase with inverted orientation (MAGI)-1/brain angiogenesis inhibitor 1 -associated protein (BAP1) as a scaffolding molecule for Rap small G protein GDP/GTP exchange protein at tight junctions. Genes Cells 5:1009-1016.

Patrie KM, Drescher AJ, Welihinda A, Mundel P, Margolis B (2002) Interaction of two actin-binding proteins, synaptopodin and alpha-actinin-4, with the tight junction protein MAGI-1. J Biol Chem 277:30183-30190.

Phillips KR, Tong S, Goodyear R, Richardson GP, Cyr JL (2006) Stereociliary myosin- $1 \mathrm{c}$ receptors are sensitive to calcium chelation and absent from cadherin 23 mutant mice. J Neurosci 26:10777-10788.

Reiners J, van Wijk E, Märker T, Zimmermann U, Jürgens K, te Brinke H, Overlack N, Roepman R, Knipper M, Kremer H, Wolfrum U (2005) Scaffold protein harmonin (USH1C) provides molecular links between Usher syndrome type 1 and type 2. Hum Mol Genet 14:3933-3943.

Sakurai A, Fukuhara S, Yamagishi A, Sako K, Kamioka Y, Masuda M, Nakaoka Y, Mochizuki N (2006) MAGI-1 is required for Rap1 activation upon cell-cell contact and for enhancement of vascular endothelial cadherin-mediated cell adhesion. Mol Biol Cell 17:966-976.

Sheng M, Sala C (2001) PDZ domains and the organization of supramolecular complexes. Annu Rev Neurosci 24:1-29.

Siemens J, Kazmierczak P, Reynolds A, Sticker M, Littlewood-Evans A, Müller U (2002) The Usher syndrome proteins cadherin 23 and harmonin form a complex by means of PDZ-domain interactions. Proc Natl Acad Sci U S A 99:14946-14951.

Siemens J, Lillo C, Dumont RA, Reynolds A, Williams DS, Gillespie PG, Müller U (2004) Cadherin 23 is a component of the tip link in hair-cell stereocilia. Nature 428:950-955.

Strochlic L, Cartaud A, Labas V, Hoch W, Rossier J, Cartaud J (2001) MAGI-1c: a synaptic MAGUK interacting with muSK at the vertebrate neuromuscular junction. J Cell Biol 153:1127-1132.

Wright GJ, Leslie JD, Ariza-McNaughton L, Lewis J (2004) Delta proteins and MAGI proteins: an interaction of Notch ligands with intracellular scaffolding molecules and its significance for zebrafish development. Development 131:5659-5669. 